\title{
Journal of Advances in Science and Engineering
} (JASE)

\section{Evaluation of net radiation using the autoregressive models with higher orders over Nigeria}

Olusola S. Ojo ${ }^{a, *}$

a Department of Physics, Federal University of Technology, Akure, Ondo State, Nigeria

\section{ARTICLE INFO}

\section{Article history:}

Received 7 May 2020

Received in revised form

26 May 2020

Accepted 27 May 2020

Available online

29 May 2020

\section{Keywords:}

Autoregressive

Climatic region

Modelling

Net radiation

Nigeria

NIMET

\begin{abstract}
In this study, monthly surface net radiation data were collected from the Nigeria Meteorological Agency, Lagos covering a duration of 31 years (1983- 2013) spatially distributed across the four climatic regions: Semi-Arid (SAR), Sub-humid Dry (SHD), Sub-humid Humid $(\mathrm{SHH})$ and Humid (HUM) regions. The net radiation was evaluated using different forms of Auto-Regressive models - AR $\{p\}$ where $p$ is the number of orders of the auto-regressive. The analysis showed that $A R \quad\{4\}$ performed best in all the regions and stations investigated. Regionally, $A R\{4\}$ has maximum values of coefficient of determination of 0.8127 in HUM, 0.7876 in SHH, 0.5765 in SHD and 0.7973 in SAR regions. It can be concluded that the higher the order of auto-regressive models, the more accurate estimation of net radiation it will give irrespective of location in Nigeria.
\end{abstract}

https://doi.org/10.37121/jase.v3i1.77

\section{Introduction}

Net radiation is the amount of the difference between upward and downward components of long wave and shortwave radiation; a central component of the Earth's equilibrium surface energy. It is the primary source of energy for the physical and chemical processes, which occur in the surfaceatmosphere interface [1]. It has served as an input parameter for the global modeling of hydrological budgets, photosynthesis, and evapotranspiration [2]. The near energy balance and precision of evapotranspiration estimation algorithms are especially affected by the net radiation [3]. For meteorology, hydrology, global change and agriculture, the accurate estimate of net radiation is critical [4]-[6]. While net radiometer can measure net radiation in a station accurately, its use is complex and time consuming for large-scale measurement, given the fact that numerous soil systems are necessary. As a result, most national weather stations focus on only small regional measurements due to economic and technological constraints that result in insufficient net radiation data for practical investigations [1].

Consequently, several scientific researchers have developed various alternative methods for estimating net radiation for multifunctional research in radiation study such as the application of satellite-derived reanalysis and analytical model of few available regional in situ data. Golkar et al. [7] investigated the net radiation estimates in Iranian arid and semi-arid climates. They evaluated multiple data sources in order to obtain a higher-spacetime-resolution net radiation estimate from the International Satellite Cloud Climatology

\footnotetext{
* Corresponding author.

Olusola S. Ojo (D) https://orcid.org/0000-0003-0958-9790 e-mail: ojoso@futa.edu.ng
}

$2636-\mathbf{6 0 7 X} /(2020$ the author. Published by Sciengtex. This is an open access article under CC BY-NC-ND license (http://creativecommons.org/licenses/by-nc-nd/4.0/) 
Project Fluxes D-series (ISCCP-FD) data set. The study concluded that global estimates, based on satellite data sets, of surface net radiation that resolve regional and weather variability, are available with reasonable precision. Krishna et al. [8] investigated the satellite-based net radiation for India. They contrasted direct computational net radiation with that of satellite data from the Cloud and Earth Radiant Energy System (CERES). It was concluded that estimated all-Sky daily net radiation was compared well with the $\mathrm{R}$ squared values of the order 0.7 and the root mean square error (RMSE) of the order 8-16 $\mathrm{W} / \mathrm{m}^{2}$. The uncertainty of surface net radiation derived from Landsat images was also studied in [9]. The paper found that there is little uncertainty between measured data and satellite data based on the root mean square analysis. The empirical assessment of daytime net radiation from shortwave and auxiliary information were studied in [10]. A new model of the day-time net radiation assessment was developed and radiation measurements were used to assess seven commonly existing models. The study found that in the globalization models, the suitable RMSE of the newly developed model is well compared.

This present work, meanwhile, applies the auto-regression models of the first, second, third and fourth orders to evaluate net radiation time series across four climatic regions in Nigeria.

\section{Materials and Methods}

\subsection{Data Source and Acquisition}

The in situ daily data of surface net radiation were collected from the Nigerian Meteorological Agency (NIMET), Lagos spanning 31 years (1983 - 2013) spatially distributed across the climatic zones in Nigeria as shown in Fig. 1. The Nigerian Meteorological Agency, initiated by an Act of the National Assembly, was enacted on $21^{\text {st }}$ of May 2003 and came into force on 19 June 2003 after the President's assents known as the NIMET (Establishment) ACT 2003. It is an agency of the government which is responsible for advising the Federal Government on all meteorological aspects; forecasting, planning, and interpreting government policies on meteorology; and making meteorological and climate forecasts on the safe operation of airplanes, oceangoing vessels, and oil facilities.

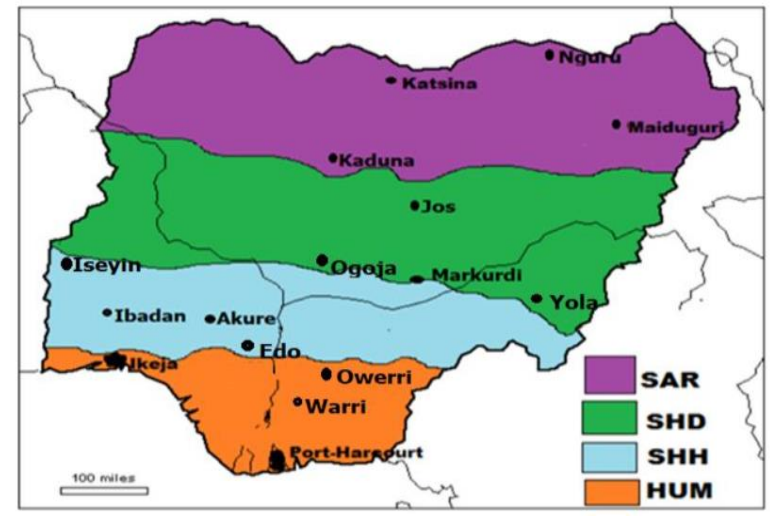

Fig. 1. Study locations in Nigeria [13].

The in-situ net radiation was used to developed the first, second, third and fourthorder autoregressive models ( $A R\{1\}, A R\{2\}$, $A R\{3\}$ and $A R\{4\}$ ) respectively using the XLSTAT 2016 edition software.

\subsection{Relevant Auto-Regressive (AR) Theory}

The AR model is a generalized model fitted to time series data either to understand the data or to estimate future points in the series based on a weighted sum of past values [11]. The dependent variable is forecasted using a linear combination of past values. AR models are generally denoted as $A R(p)$ where parameters $p$ is the order (number of time lags) of the autoregressive model [12]. The general expression is given by:

$$
y_{t}=\alpha+\emptyset_{1} y_{t-1}+\emptyset_{2} y_{t-2}+\cdots+\emptyset_{p} y_{t-p}+\varepsilon_{t},
$$

where $\emptyset_{p}$ is the parameter estimate of autoregressive $p^{\text {th }}$ order, $\mathrm{a}$ is the constant, $\varepsilon \mathrm{t}$ is error term and $y_{t}$ is the estimated variable.

AR parameters can be obtained using the least-squares approach, which can be written in compact form on k-step ahead prediction for $\mathrm{p}^{\text {th }}$ order AR process as [14], [15]:

$$
\hat{Y}=U \hat{\beta},
$$

where,

$$
\begin{aligned}
\hat{Y} & =\left[\begin{array}{c}
y_{t} \\
y_{t+1} \\
\vdots \\
y_{t+k}
\end{array}\right], \\
U & =\left[\begin{array}{cccc}
y_{t-1} & y_{t-2} & \vdots & y_{t-p} \\
y_{t} & y_{t-1} & \vdots & y_{t+1-p} \\
\vdots & \vdots & \vdots & \vdots \\
y_{t+k-1} & y_{t+k-2} & \cdots & y_{t+k-p}
\end{array}\right] \\
\hat{\beta} & =\left[\begin{array}{c}
\emptyset_{1} \\
\emptyset_{2} \\
\vdots \\
\emptyset_{p}
\end{array}\right]
\end{aligned}
$$

Using the matrix notation, AR parameter $\hat{\beta}$ can be evaluated as:

$$
\hat{\beta}=\left(U^{T} U\right)^{-1} U^{T} \hat{Y}
$$


In this paper, the monthly data sets used were sub-divided into two such that the first data set A consist of twenty-five years (1983 -2007) data and data set $B$ consists of six years (2008 - 2013) data. The first data set was used for development of $A R(p)$ models while the second part was used for evaluation of the performance of the model following the method described in [16].

\subsection{Performance Evaluation of $A R(p)$ Model}

The performance of the $A R(p)$ model developed in this study was assessed using the mean squared error (MSE), root mean squared error, coefficient of determination $\left(R^{2}\right)$, mean absolute percentage error (MAPE) and Akaike's information criterion (AIC) as expressed in Equations (5) to (9). Several studies used $A R(p)$ techniques to evaluate the global solar radiation [17]-[20], total ozone content [21] and ocean current [22]. The method was found to predict the variables very well with great accuracy compared to ground measurement.

$$
\begin{aligned}
& M S E=\frac{1}{n} \sum_{n=1}^{n}\left(P_{i}-\mathrm{O}_{i}\right)^{2}, \\
& R M S E=\sqrt{\frac{1}{n} \sum_{n=1}^{n}\left(P_{i}-\mathrm{O}_{i}\right)^{2},} \\
& \mathrm{R}^{2}=\left(\frac{\mathrm{n}\left(\sum \mathrm{P}_{i} \mathrm{O}_{i}\right)-\left(\sum \mathrm{P}_{i}\right)\left(\sum \mathrm{O}_{i}\right)}{\sqrt{\left(\mathrm{n} \sum \mathrm{o}_{i}^{2}\right)-\left(\sum \mathrm{o}_{i}{ }^{2}\right)\left[\left(\mathrm{n} \sum \mathrm{P}_{i}^{2}\right)-\left(\sum \mathrm{P}_{i}^{2}\right)\right]}}\right)^{2}, \\
& M A P E=\frac{1}{n} \sum_{n=1}^{n}\left|\frac{P_{i}-\mathrm{O}_{i}}{\mathrm{O}_{i}}\right|, \\
& \mathrm{AIC}=-2(\text { Log - likelihood })+2 \mathrm{~K},
\end{aligned}
$$

where $O_{i}$ is the observed values and $P_{i}$ is the predicted values, $n$ is the number of observations, $K$ is the number of model parameters (the number of variables in the model plus the intercept) and Log-likelihood is a measure of model fit.

For the purpose of error analysis, the MSE and RMSE is a measure of the quality of an estimator; it is always non-negative, and values closer to zero are better [23]-[24]. The $\mathrm{R}^{2}$ is the proportion of the variance in the dependent variable, which can be estimated from the independent variables. Its value normally ranges from 0 to 1 and the closer to unity is desirable [12], [25]. The AIC index compares the quality of a set of statistical models to each other. The lower the value, the better the model but it does not say anything about the absolute quality of the model [17], [26].

\section{Results and Discussion}

\subsection{Developed AR Models}

Tables 1 - 4 show the parameter estimates of four different $A R\{p\}$ order models over the four climatic regions and some selected stations in each of the regions. These values formed models with lag numbers that can be used to evaluate net radiation in each of regions and their respective stations over Nigeria. For instance, in the entire Semi-Arid region, the first, second, third and fourthorder autoregressive models of the form shown in Equations (10) to (13) respectively were formed from the parameter estimates presented in Table 1.

Table 1 Model parameter of the ARMA $(p, q)$ model for Semi-Arid (SAR) Region

\begin{tabular}{ccccccc}
\hline \hline \multirow{2}{*}{ Stations } & Model & \multicolumn{5}{c}{ Parameter } \\
\cline { 3 - 7 } & & $\mathrm{C}$ & $\emptyset_{1}$ & $\emptyset_{2}$ & $\emptyset_{3}$ & $\emptyset_{4}$ \\
\hline \hline \multirow{3}{*}{ Semi-Arid } & ARMA $(1,0)$ & 98.7498 & 0.7866 & - & - & - \\
& ARMA $(2,0)$ & 99.2495 & 1.2619 & -0.6100 & - & - \\
& ARMA $(3,0)$ & 99.3007 & 1.1295 & -0.3356 & -0.2183 & - \\
& ARMA $(4,0)$ & 99.3268 & 1.0544 & -0.4500 & 0.1713 & -0.3470 \\
\hline \multirow{3}{*}{ Maiduguri } & ARMA $(1,0)$ & 71.1822 & 0.7925 & - & - & - \\
& ARMA $(2,0)$ & 71.4069 & 1.1864 & -0.4960 & - & - \\
& ARMA $(3,0)$ & 71.4524 & 0.9744 & 0.0111 & -0.4260 & - \\
& ARMA $(4,0)$ & 71.4391 & 0.8302 & 0.0165 & -0.0987 & -0.3358 \\
\hline \multirow{5}{*}{ Kastina } & ARMA $(1,0)$ & 94.4578 & 0.8154 & - & - & - \\
& ARMA $(2,0)$ & 95.2160 & 1.3837 & -0.6989 & - & - \\
& ARMA $(3,0)$ & 95.2726 & 1.1097 & -0.1565 & -0.3906 & - \\
& ARMA $(4,0)$ & 95.2750 & 1.0170 & -0.1962 & -0.1221 & -0.2402 \\
\hline \multirow{5}{*}{ Kaduna } & ARMA $(1,0)$ & 113.2804 & 0.6383 & - & - & - \\
& ARMA $(2,0)$ & 113.6051 & 0.9932 & -0.5680 & - & - \\
& ARMA $(3,0)$ & 113.6067 & 0.9901 & -0.5625 & -0.0056 & - \\
& ARMA $(4,0)$ & 113.6337 & 0.9892 & -0.6266 & 0.1067 & -0.1155 \\
\hline \multirow{5}{*}{ Nguru } & ARMA $(1,0)$ & 116.4764 & 0.7232 & - & - & - \\
& ARMA $(2,0)$ & 116.9072 & 1.1325 & -0.5743 & - & - \\
& ARMA $(3,0)$ & 116.9208 & 1.1119 & -0.5339 & -0.0360 & - \\
& ARMA $(4,0)$ & 116.9664 & 1.1049 & -0.6565 & 0.2178 & -0.2300 \\
\hline \hline
\end{tabular}


Table 2 Model parameter of the ARMA $(p, q)$ model for Sub-humid Dry (SHD) Region

\begin{tabular}{|c|c|c|c|c|c|c|}
\hline \multirow[t]{2}{*}{ Stations } & \multirow[t]{2}{*}{ Model } & \multicolumn{5}{|c|}{ Parameter } \\
\hline & & $\mathrm{C}$ & $\emptyset_{1}$ & $\emptyset_{2}$ & $\emptyset_{3}$ & $\emptyset_{4}$ \\
\hline \multirow{4}{*}{ SHD } & ARMA $(1,0)$ & 125.8921 & 0.4997 & - & - & - \\
\hline & $\operatorname{ARMA}(2,0)$ & 126.0284 & 0.8177 & -0.6535 & - & - \\
\hline & ARMA $(3,0)$ & 126.0472 & 0.7225 & -0.5383 & -0.1435 & - \\
\hline & ARMA $(4,0)$ & 126.0373 & 0.7359 & -0.4834 & -0.2145 & 0.0997 \\
\hline \multirow{4}{*}{ Jos } & ARMA $(1,0)$ & 115.3656 & 0.5679 & - & - & - \\
\hline & $\operatorname{ARMA}(2,0)$ & 115.5692 & 0.9241 & -0.6386 & - & - \\
\hline & ARMA $(3,0)$ & 115.5770 & 0.8998 & -0.6040 & -0.0379 & - \\
\hline & $\operatorname{ARMA}(4,0)$ & 115.5749 & 0.9002 & -0.5962 & -0.0493 & 0.0129 \\
\hline \multirow{4}{*}{ Yola } & ARMA $(1,0)$ & 119.1401 & 0.5755 & - & - & - \\
\hline & $\operatorname{ARMA}(2,0)$ & 119.3792 & 0.8749 & -0.5368 & - & - \\
\hline & ARMA $(3,0)$ & 119.3910 & 0.8450 & -0.4884 & -0.0561 & - \\
\hline & ARMA $(4,0)$ & 119.3893 & 0.8456 & -0.4830 & -0.0656 & 0.0114 \\
\hline \multirow{4}{*}{ Makurdi } & ARMA $(1,0)$ & 143.1686 & 0.7003 & - & - & - \\
\hline & $\operatorname{ARMA}(2,0)$ & 143.2292 & 0.9824 & -0.4016 & - & - \\
\hline & ARMA $(3,0)$ & 143.0801 & 0.8456 & -0.0760 & -0.3110 & - \\
\hline & ARMA $(4,0)$ & 143.0812 & 0.8136 & -0.0899 & -0.2133 & -0.1110 \\
\hline \multirow{4}{*}{ Ogoja } & ARMA $(1,0)$ & 144.0111 & 0.7744 & - & - & - \\
\hline & ARMA $(2,0)$ & 143.8506 & 1.0914 & -0.4112 & - & - \\
\hline & ARMA $(3,0)$ & 143.8952 & 0.8774 & 0.1390 & -0.4978 & - \\
\hline & ARMA $(4,0)$ & 143.8944 & 0.7559 & 0.1585 & -0.2589 & -0.2604 \\
\hline
\end{tabular}

Table 3 Model parameter of the ARMA $(p, q)$ model for Sub-humid Humid (SHH) Region

\begin{tabular}{|c|c|c|c|c|c|c|}
\hline \multirow[t]{2}{*}{ Stations } & \multirow[t]{2}{*}{ Model } & \multicolumn{5}{|c|}{ Parameter } \\
\hline & & $\mathrm{C}$ & $\emptyset_{1}$ & $\emptyset_{2}$ & $\emptyset_{3}$ & $\emptyset_{4}$ \\
\hline \multirow{4}{*}{$\mathrm{SHH}$} & ARMA $(1,0)$ & 128.6180 & 0.7916 & - & - & - \\
\hline & ARMA $(2,0)$ & 128.4837 & 1.2506 & -0.5826 & - & - \\
\hline & ARMA $(3,0)$ & 128.5146 & 1.0395 & -0.1327 & -0.3596 & - \\
\hline & $\operatorname{ARMA}(4,0)$ & 128.5148 & 0.9978 & -0.1502 & -0.2357 & -0.1182 \\
\hline \multirow{4}{*}{ Ibadan } & $\operatorname{ARMA}(1,0)$ & 125.1214 & 0.8038 & - & - & - \\
\hline & ARMA $(2,0)$ & 124.9540 & 1.2958 & -0.6161 & - & - \\
\hline & ARMA $(3,0)$ & 124.9818 & 1.0830 & -0.1700 & -0.3448 & - \\
\hline & ARMA $(4,0)$ & 124.9842 & 1.0234 & -0.2008 & -0.1558 & -0.1744 \\
\hline \multirow{4}{*}{ Iseyin } & ARMA $(1,0)$ & 136.3767 & 0.7589 & - & - & - \\
\hline & ARMA $(2,0)$ & 136.3536 & 1.2055 & -0.5876 & - & - \\
\hline & ARMA $(3,0)$ & 136.3711 & 1.1017 & -0.3755 & -0.1758 & - \\
\hline & ARMA $(4,0)$ & 136.3784 & 1.0877 & -0.4053 & -0.0889 & -0.0790 \\
\hline \multirow{4}{*}{ Edo } & ARMA $(1,0)$ & 119.8012 & 0.7916 & - & - & - \\
\hline & ARMA $(2,0)$ & 119.5801 & 1.1858 & -0.5026 & - & - \\
\hline & ARMA $(3,0)$ & 119.5932 & 0.9570 & 0.0323 & -0.4509 & - \\
\hline & $\operatorname{ARMA}(4,0)$ & 119.5727 & 0.8327 & 0.0338 & -0.1745 & -0.2843 \\
\hline \multirow{4}{*}{ Akure } & ARMA $(1,0)$ & 133.1837 & 0.7598 & - & - & - \\
\hline & ARMA $(2,0)$ & 133.0980 & 1.1086 & -0.4611 & - & - \\
\hline & ARMA $(3,0)$ & 133.1271 & 0.9294 & -0.0353 & -0.3838 & - \\
\hline & ARMA $(4,0)$ & 133.1250 & 0.8862 & -0.0415 & -0.2752 & -0.1154 \\
\hline
\end{tabular}

These can be applied to estimate net radiation in the Semi-Arid region.

$$
\begin{aligned}
\mathrm{Q}_{t}=98.7498+0.7866 \mathrm{Q}_{t-1} \\
\mathrm{Q}_{t}=99.2495+1.2619 \mathrm{Q}_{t-1}-0.61 \mathrm{Q}_{t-2} \\
\mathrm{Q}_{t}=99.3007+1.1295 \mathrm{Q}_{t-1}-0.3356 \mathrm{Q}_{t-2} \\
\quad-0.2183 \mathrm{Q}_{t-3} \\
\mathrm{Q}_{t}=99.3268+1.0544 \mathrm{Q}_{t-1}-0.45 \mathrm{Q}_{t-2} \\
\quad+0.1713 \mathrm{Q}_{t-3}-0.3470 \mathrm{Q}_{t-4}
\end{aligned}
$$

Also, for the entire Sub-humid Dry (SHD) region, the equations of the form shown in Equations (14) to (17) can be applied to estimate net radiation as shown in Table 2.

$$
\begin{aligned}
& Q_{t}=125.8921+0.4997 Q_{t-1} \\
& Q_{t}=126.0284+0.8177 Q_{t-1}-0.6535 Q_{t-2} \\
& Q_{t}=126.0472+0.7225 Q_{t-1}-0.5383 Q_{t-2} \\
&-0.1435 Q_{t-3} \\
& Q_{t}=126.0373+0.7359 Q_{t-1}-0.4834 Q_{t-2} \\
& \quad+0.2145 Q_{t-3}-0.0997 Q_{t-4}
\end{aligned}
$$


Table 4 Model parameter of the ARMA $(p, q)$ model for Humid (HUM) Region

\begin{tabular}{|c|c|c|c|c|c|c|}
\hline \multirow[t]{2}{*}{ Stations } & \multirow[t]{2}{*}{ Model } & \multicolumn{5}{|c|}{ Parameter } \\
\hline & & $\mathrm{C}$ & $\emptyset_{1}$ & $\emptyset_{2}$ & $\emptyset_{3}$ & $\emptyset_{4}$ \\
\hline \multirow{4}{*}{ Humid } & ARMA $(1,0)$ & 131.0781 & 0.8002 & - & - & - \\
\hline & ARMA $(2,0)$ & 130.8208 & 1.2291 & -0.5382 & - & - \\
\hline & ARMA $(3,0)$ & 130.8547 & 0.9816 & 0.0192 & -0.4495 & - \\
\hline & ARMA $(4,0)$ & 130.8760 & 0.8351 & 0.0180 & -0.1191 & -0.3313 \\
\hline \multirow{4}{*}{$\begin{array}{c}\text { Port } \\
\text { Harcourt }\end{array}$} & ARMA $(1,0)$ & 125.7165 & 0.8022 & - & - & - \\
\hline & ARMA $(2,0)$ & 125.2572 & 1.2239 & -0.5287 & - & - \\
\hline & ARMA $(3,0)$ & 125.2444 & 1.0090 & -0.0351 & -0.4000 & - \\
\hline & ARMA $(4,0)$ & 125.2626 & 0.8399 & -0.0499 & 0.0229 & -0.4174 \\
\hline \multirow{4}{*}{ Warri } & ARMA $(1,0)$ & 130.3512 & 0.7232 & - & - & - \\
\hline & ARMA $(2,0)$ & 130.2431 & 0.9861 & -0.3643 & - & - \\
\hline & ARMA $(3,0)$ & 130.2328 & 0.8721 & -0.0583 & -0.3093 & - \\
\hline & ARMA $(4,0)$ & 130.2484 & 0.7772 & -0.0748 & -0.0474 & -0.3001 \\
\hline \multirow{4}{*}{ Owerri } & ARMA $(1,0)$ & 130.8932 & 0.8047 & - & - & - \\
\hline & ARMA $(2,0)$ & 130.5314 & 1.2229 & -0.5223 & - & - \\
\hline & ARMA $(3,0)$ & 130.5638 & 0.9718 & 0.0573 & -0.4681 & - \\
\hline & ARMA $(4,0)$ & 130.5954 & 0.7861 & 0.0707 & -0.0690 & -0.4030 \\
\hline \multirow{4}{*}{ Ikeja } & ARMA $(1,0)$ & 124.4185 & 0.7408 & - & - & - \\
\hline & ARMA $(2,0)$ & 124.3811 & 1.0949 & -0.4793 & - & - \\
\hline & ARMA $(3,0)$ & 124.4039 & 0.9594 & -0.1718 & -0.2815 & - \\
\hline & ARMA $(4,0)$ & 124.4142 & 0.9083 & -0.2033 & -0.1074 & -0.1819 \\
\hline
\end{tabular}

In the same vein, for the entire Sub-humid Humid region, the equations of the form shown in (18)-(21) can be applied to estimate net radiation as presented in Table 3 .

$$
\begin{aligned}
\mathrm{Q}_{t} & =128.6180+0.7916 \mathrm{Q}_{t-1} \\
\mathrm{Q}_{t} & =128.4837+1.2506 \mathrm{Q}_{t-1}-0.5826 \mathrm{Q}_{t-2} \\
\mathrm{Q}_{t} & =128.5146+1.0395 \mathrm{Q}_{t-1}-0.1327 \mathrm{Q}_{t-2} \\
& -0.3596 \mathrm{Q}_{t-3} \\
\mathrm{Q}_{t} & =128.5148+0.9978 \mathrm{Q}_{t-1}-0.1502 \mathrm{Q}_{t-2} \\
\quad & +0.2357 \mathrm{Q}_{t-3}-0.1182 \mathrm{Q}_{t-4}
\end{aligned}
$$

Finally, for the entire Humid region, the equations of the form shown in (22) - (25) can be applied to estimate net radiation as presented in Table 4.

$$
\begin{aligned}
Q_{t}=131.0781+0.8002 Q_{t-1} \\
Q_{t}=130.8208+1.2291 Q_{t-1}-0.5382 Q_{t-2} \\
Q_{t}=130.8547+0.9816 Q_{t-1}+0.0192 Q_{t-2} \\
\quad-0.4495 Q_{t-3} \\
Q_{t}=130.8760+0.8351 Q_{t-1}+0.0180 Q_{t-2} \\
\quad-0.1191 Q_{t-3}-0.3313 Q_{t-4}
\end{aligned}
$$

Those of other stations in the SAR, SHD, $\mathrm{SHH}$ and Humid region can be formed in the same manner. Those set of equations were used to estimate and predict net radiation in the stations within the respective region as shown in Table 5 a.

\subsection{Assessment and Validation of AR Models}

Table 5 shows the result of the application of the $A R\{p\}$ models over Nigeria. From the table, it can be observed that the estimated net radiation from each of the four $A R\{p\}$ equations were very close to the observed values with differences less than $3.50 \mathrm{~W} / \mathrm{m}^{2}$ in all regions and their respective stations. The $\mathrm{R}$-squared values were found to be more than 0.30 threshold value, proposed for the suitability of the model in [27]. The only exception is the value of the AR $\{1\}$ model in the Sub-humid Dry with a value of 0.2455 . These good fits also attest to the significance of all other statistical indicators. It can also be established from Table 5 that the higher the orders of the $\operatorname{AR}\{p\}$, the better the performance of the model. Therefore, $\operatorname{AR}\{4\}$ showed the best performance in all the stations and the four regions. That is, it has the highest values of $\mathrm{R}^{2}$ (Table $5 \mathrm{a}$ ) and lowest values of MSE, RMSE, MAPE and AIC (Table $5 b)$.

\subsection{Comparison of Observed and Predicted Net Radiation over Nigeria.}

Figs. 2 - 5 show the scatter plots of comparison between observed and $\operatorname{AR}\{\mathrm{p}\}$ net radiation over four climatic regions in Nigeria. The observations show that the autoregressive model of order $4(A R\{4\})$ has the highest $R^{2}$ values in the SAR, SHD and HUM regions having magnitudes of $0.7973,0.5765$ and 0.8127 respectively but $A R\{2\}$ has the highest value in $\mathrm{SHH}$ region having a magnitude of 0.8011 . The $A R\{p\}$ performed best in the Humid region followed by $\mathrm{SHH}$ but it has the worst performance in the SHD region. 
Table 5a Assessment of observed and predicted net radiation over sixteen stations across four regions in Nigeria using the AR ( $p$ ) Model

\begin{tabular}{|c|c|c|c|c|c|c|c|c|c|c|c|c|c|}
\hline \multirow[b]{3}{*}{ Stations } & \multicolumn{5}{|c|}{ Net Radiation } & \multicolumn{8}{|c|}{ AR (p) Models Test Parameters } \\
\hline & \multirow[b]{2}{*}{ Observed } & \multirow[b]{2}{*}{$\operatorname{AR}\{1\}$} & \multirow[b]{2}{*}{$\mathrm{AR}\{2\}$} & \multirow[b]{2}{*}{$\operatorname{AR}\{3\}$} & \multirow[b]{2}{*}{$\operatorname{AR}\{4\}$} & \multicolumn{4}{|c|}{$\mathrm{R}^{2}$} & \multicolumn{4}{|c|}{ MSE } \\
\hline & & & & & & $\operatorname{AR}\{1\}$ & $\operatorname{AR}\{2\}$ & $\operatorname{AR}\{3\}$ & $\operatorname{AR}\{4\}$ & $\operatorname{AR}\{1\}$ & $\operatorname{AR}\{2\}$ & $\operatorname{AR}\{3\}$ & $\mathrm{AR}\{4\}$ \\
\hline SAR & 101.0236 & 97.5434 & 99.3882 & 99.5847 & 99.7422 & 0.6154 & 0.7579 & 0.7694 & 0.7973 & 100.4789 & 63.2570 & 60.2370 & 52.9561 \\
\hline Maiduguri & 76.9432 & 70.6348 & 71.6000 & 71.8030 & 71.7859 & 0.6307 & 0.7217 & 0.7725 & 0.7983 & 89.3172 & 67.3146 & 55.0205 & 48.7823 \\
\hline Kastina & 97.8225 & 93.0589 & 95.8129 & 95.9048 & 95.9093 & 6653 & 0.8284 & 0.8544 & 0.8628 & 197.6089 & 101.2884 & 85.9319 & 913 \\
\hline Kaduna & 114.2277 & 112.5326 & 113.6891 & 113.6962 & 113.8362 & 0.4021 & 0.5932 & 0.5932 & 0.5986 & 142.9692 & 97.2698 & 97.2668 & 801 \\
\hline Nguru & 115.1008 & 115.3494 & 116.9107 & 116.9637 & 117.1964 & 0.5172 & 0.6753 & 0.6758 & 0.6929 & 137.1871 & 92.2429 & 92.1236 & 87.2661 \\
\hline$\overline{S H D}$ & 124.0316 & 125.5812 & 126.1390 & 126.2370 & 126.1750 & .2455 & 0.5641 & 0.5725 & 0.5765 & 62.8 & 17 & 94 & 75 \\
\hline Jos & 115 & 114.9809 & 115 & 115 & & & & & & & & & 85 \\
\hline Yola & 114.1320 & 118.5987 & 119.4461 & 119.4967 & 84 & 51 & 167 & 0.5182 & 83 & 106.6166 & 15 & 39 & 042 \\
\hline Makurdi & & 143.0419 & 143.5310 & & & & & & & & & & \\
\hline Ogoja & 144.8967 & 143.0435 & 143.2020 & 143.8948 & 143.9993 & 0.5952 & 0.6627 & & 0.7586 & 238 & 90.1687 & 753 & 73 \\
\hline$\overline{\mathrm{SHH}}$ & 130.7683 & 127.8657 & 128.3438 & 128.6889 & 128.7319 & 0.6258 & 0.7530 & 0.7847 & 0.7876 & 99.9853 & 65.9885 & 57.5322 & 56.7324 \\
\hline Ibadan & & 124.3741 & 124.9563 & 125.2767 & 125.3616 & & & & & & & & 12 \\
\hline Iseyin & 139.3202 & 135.9300 & 136.4060 & 136.5531 & 136.6103 & 0.5784 & 0.7247 & 0.7332 & 0.7349 & 110.0616 & 71.8725 & 69.6462 & 880 \\
\hline Edo & 120.9932 & 118.9285 & 119.1999 & 119.6428 & 119.6736 & 0.6217 & 0.7167 & 0.7734 & 0.7914 & 111.0305 & 83.1139 & 66.4877 & 61.1944 \\
\hline Akure & 135.7472 & 132.4298 & 132.8329 & 133.2394 & 133.2699 & 0.5765 & 0.6666 & 0.7153 & 0.7191 & 116.5678 & 91.7744 & 78.3662 & 77.3294 \\
\hline HUM & 132.8384 & 130.1367 & 130.1813 & 130.6790 & 130.9010 & & 0.7405 & 0.7907 & 0.8127 & & 71.1040 & 57.3518 & 51.3144 \\
\hline Port-Harcourt & 129.0480 & 125.1225 & 124.5091 & 124.8576 & 125.2098 & 0.6384 & 0.7376 & 0.7777 & 0.8144 & 135.8628 & 98.5713 & 83.5075 & 69.7273 \\
\hline Warri & 130.9401 & 129.4809 & 129.6362 & 129.8636 & 130.0939 & 0.5201 & 0.5829 & 0.6223 & 0.6559 & 147.4002 & 128.0920 & 115.9926 & 105.6726 \\
\hline Owerri & 133.5132 & 129.8945 & 129.6703 & 130.2488 & 130.5877 & 0.6423 & 0.7377 & 0.7917 & 0.8234 & 121.2313 & 88.8603 & 70.5727 & 59.8478 \\
\hline Ikeja & 125.7941 & 123.9288 & 124.3313 & 124.5831 & 124.6876 & 0.5499 & 0.6534 & 0.6807 & 0.6913 & 91.0370 & 70.1057 & 64.5733 & 62.4323 \\
\hline
\end{tabular}


Table 5b Assessment of observed and predicted net radiation over sixteen stations across four regions in Nigeria using the AR ( $p$ ) Model

\begin{tabular}{|c|c|c|c|c|c|c|c|c|c|c|c|c|}
\hline \multirow[b]{3}{*}{ Stations } & \multicolumn{12}{|c|}{ AR (p) Models Test Parameters } \\
\hline & \multicolumn{4}{|c|}{ RMSE } & \multicolumn{4}{|c|}{ MAPE } & \multicolumn{4}{|c|}{ AIC } \\
\hline & $\mathrm{AR}\{1\}$ & $\operatorname{AR}\{2\}$ & $\mathrm{AR}\{3\}$ & $\operatorname{AR}\{4\}$ & $\mathrm{AR}\{1\}$ & $\operatorname{AR}\{2\}$ & $\operatorname{AR}\{3\}$ & $\operatorname{AR}\{4\}$ & $\operatorname{AR}\{1\}$ & $\operatorname{AR}\{2\}$ & $\operatorname{AR}\{3\}$ & $\operatorname{AR}\{4\}$ \\
\hline SAR & 10.0239 & 7.9534 & 7.7612 & 7.2771 & 8.7564 & 6.4965 & 6.2047 & 5.8940 & 2239.3117 & 2103.4086 & 2090.8841 & 2054.7710 \\
\hline Maiduguri & 9.4508 & 8.2045 & 7.4176 & 6.9844 & 11.5184 & 9.7056 & 8.5708 & 8.0134 & 2204.0104 & 2121.7324 & 2063.8262 & 2030.1912 \\
\hline Kastina & 14.0573 & 10.0642 & 9.2699 & 8.9995 & 13.3470 & 8.4581 & 8.0795 & 8.0517 & 2442.3434 & 2245.1844 & 2198.3417 & 2182.8030 \\
\hline Kaduna & 11.9570 & 9.8625 & 9.8624 & 9.7969 & 9.2490 & 7.3002 & 7.3008 & 7.2429 & 2344.6753 & 2231.9017 & 2233.8925 & 2231.9558 \\
\hline Nguru & 11.7127 & 9.6043 & 9.5981 & 9.3416 & 8.6447 & 6.7378 & 6.7197 & 6.5480 & 2332.5072 & 2216.2198 & 2217.8366 & 2203.8153 \\
\hline SHD & 7.9299 & 6.0276 & 5.9690 & 5.9412 & 5.2435 & 3.7288 & 3.6726 & 3.6879 & 2098.0320 & 1936.5648 & 1932.7781 & 1931.9987 \\
\hline Jos & 9.8275 & 7.5801 & 7.5748 & 7.5742 & 7.2381 & 5.3812 & 5.3755 & 5.3763 & 2226.8649 & 2074.1135 & 2075.6963 & 2077.6481 \\
\hline Yola & 10.3255 & 8.7379 & 8.7243 & 8.7238 & 7.3064 & 6.0156 & 6.0124 & 6.0079 & 2256.5370 & 2159.0388 & 2160.1153 & 2162.0773 \\
\hline Makurdi & 9.9927 & 9.1608 & 8.8269 & 8.7742 & 5.7254 & 5.2178 & 5.0394 & 5.0052 & 2237.1530 & 2187.3539 & 2167.3283 & 2165.7827 \\
\hline Ogoja & 10.4031 & 9.4957 & 8.3051 & 8.0323 & 6.0406 & 5.3095 & 4.5643 & 4.3756 & 2261.5392 & 2209.1498 & 2131.5803 & 2113.7952 \\
\hline $\mathrm{SHH}$ & 9.9993 & 8.1233 & 7.5850 & 7.5321 & 6.5325 & 5.1469 & 4.7764 & 4.7141 & 2237.8551 & 2116.0160 & 2077.2842 & 2075.1399 \\
\hline Ibadan & 10.0135 & 7.8866 & 7.4065 & 7.2932 & 6.7503 & 5.1857 & 4.8494 & 4.6991 & 2238.7637 & 2098.4438 & 2063.1459 & 2056.0243 \\
\hline Iseyin & 10.4910 & 8.4778 & 8.3454 & 8.3191 & 6.3959 & 4.9865 & 4.8467 & 4.8390 & 2266.5329 & 2141.5378 & 2134.1906 & 2134.3222 \\
\hline Edo & 10.5371 & 9.1167 & 8.1540 & 7.8227 & 7.5310 & 6.3119 & 5.6283 & 5.3352 & 2269.2896 & 2184.9840 & 2120.6989 & 2098.1449 \\
\hline Akure & 10.7967 & 9.5799 & 8.8525 & 8.7937 & 6.6904 & 5.8979 & 5.3513 & 5.3142 & 2283.7661 & 2214.4986 & 2169.5871 & 2167.6433 \\
\hline HUM & 9.9797 & 8.4323 & 7.5731 & 7.1634 & 6.3980 & 5.2682 & 4.7036 & 4.4158 & 2236.7192 & 2138.3074 & 2076.4684 & 2045.5381 \\
\hline Port-Harcourt & 11.6560 & 9.9283 & 9.1382 & 8.3503 & 7.6470 & 6.3645 & 5.8910 & 5.4161 & 2329.8886 & 2236.2771 & 2189.0260 & 2137.6532 \\
\hline Warri & 12.1408 & 11.3178 & 10.7700 & 10.2797 & 7.9178 & 7.3193 & 6.8836 & 6.3728 & 2354.0489 & 2314.2118 & 2286.7424 & 2261.1557 \\
\hline Owerri & 11.0105 & 9.4266 & 8.4008 & 7.7361 & 7.0275 & 5.8778 & 5.1744 & 4.8436 & 2295.7158 & 2205.1566 & 2138.7284 & 2091.9397 \\
\hline Ikeja & 9.5413 & 8.3729 & 8.0358 & 7.9014 & 6.1970 & 5.4765 & 5.2600 & 5.0911 & 2209.5388 & 2133.6801 & 2111.2665 & 2103.2883 \\
\hline
\end{tabular}


(a) Semi Arid - AR $\{\mathbf{I}\}$

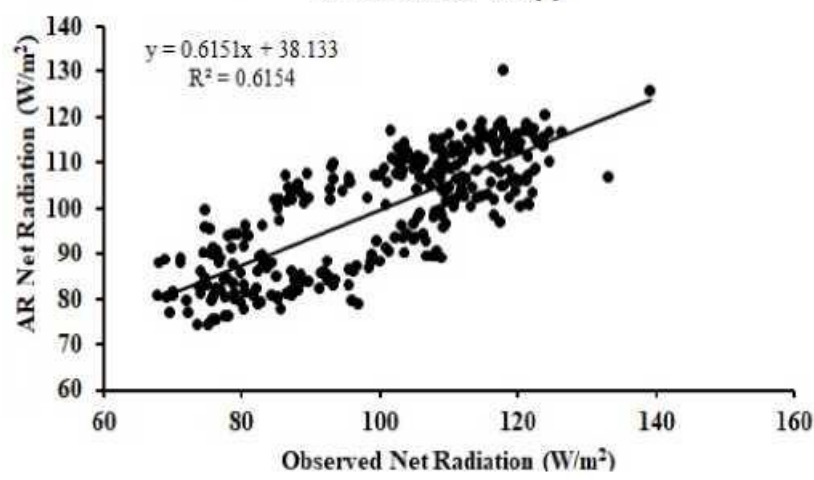

(c) Semi Arid - AR \{3\}

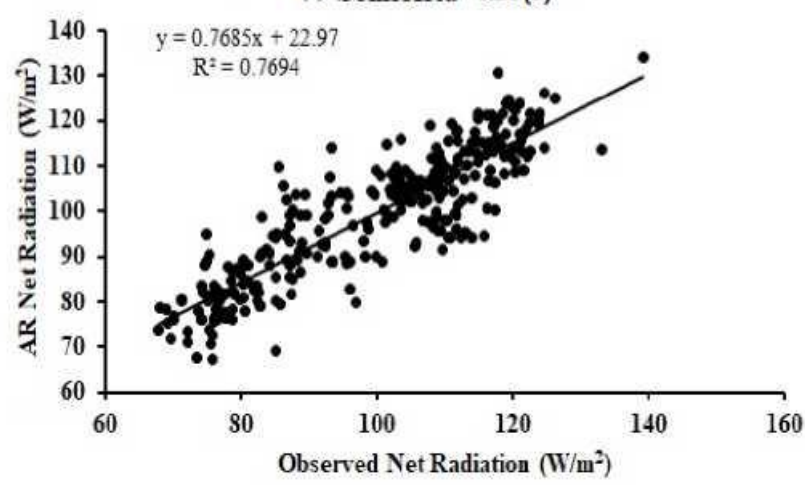

(b) Semi Arid - AR $\{2\}$

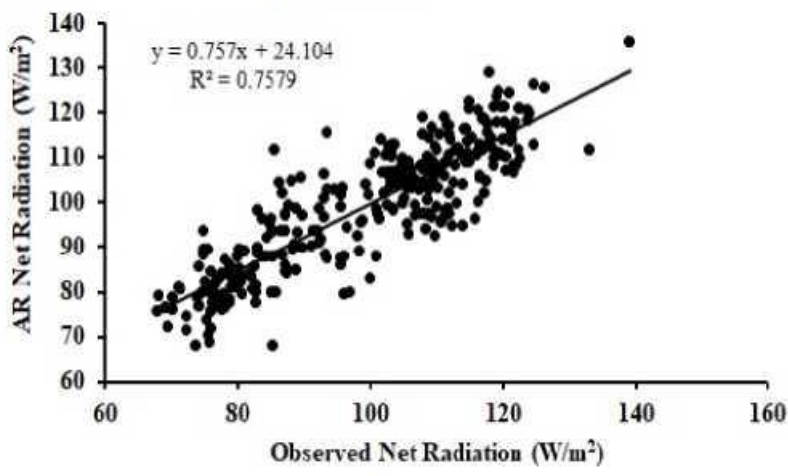

(d) Semi Arid - AR \{4\}

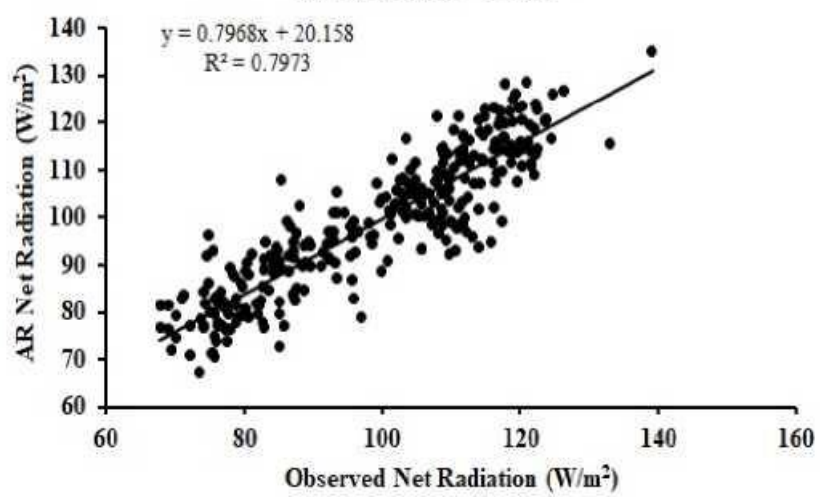

Fig. 2 Scatter plots of the observed net radiation and AR net radiation over SAR zone in Nigeria

(a) $\mathrm{SHD}-\mathrm{AR}\{1\}$

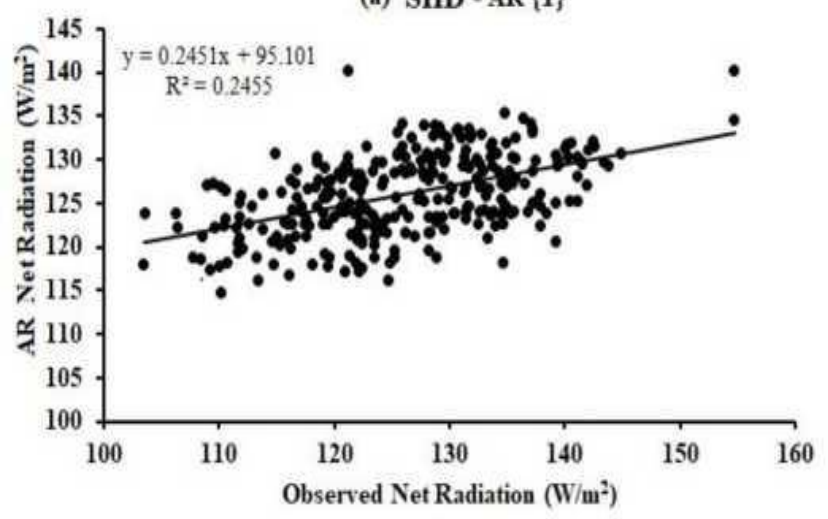

(c) SHD - AR \{3\}

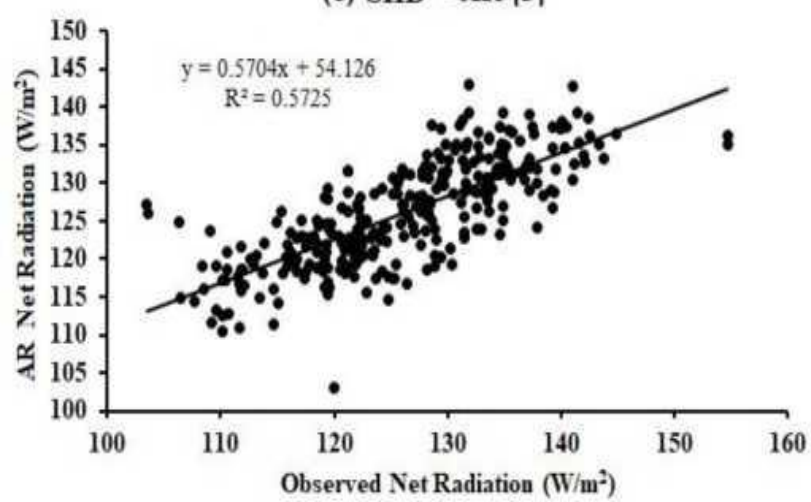

(b) SHD - AR $\{2\}$

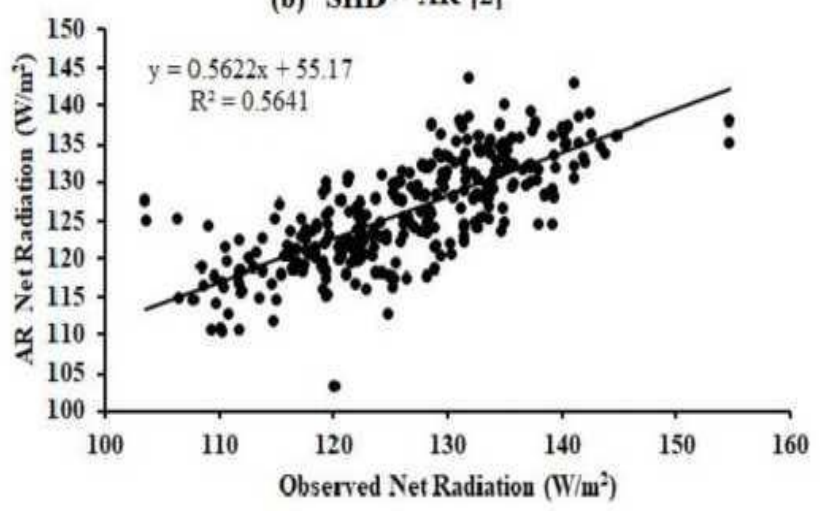

(d) SHD - AR $\{4\}$

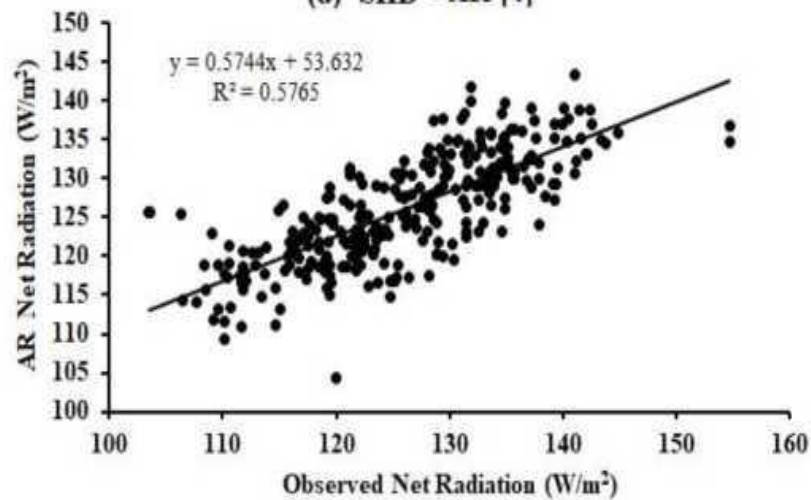

Fig. 3 Scatter plots of the observed net radiation and AR net radiation over SHD zone in Nigeria 
(a) SHH - AR $\{1\}$

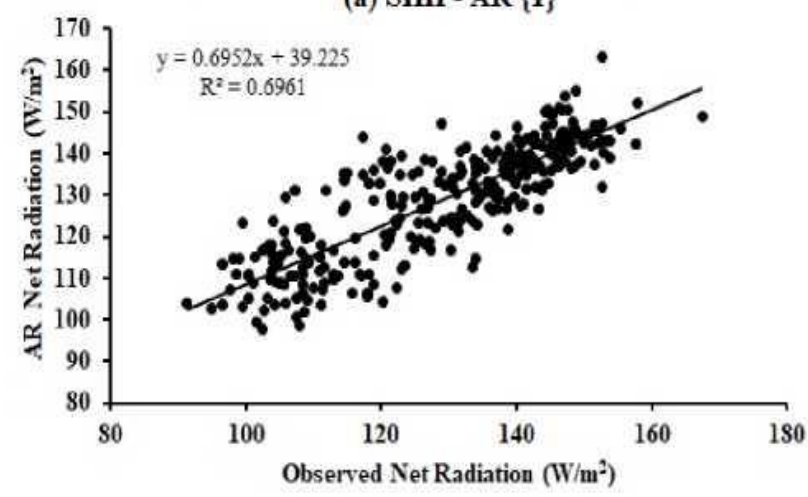

(c) SHH - AR \{3\}

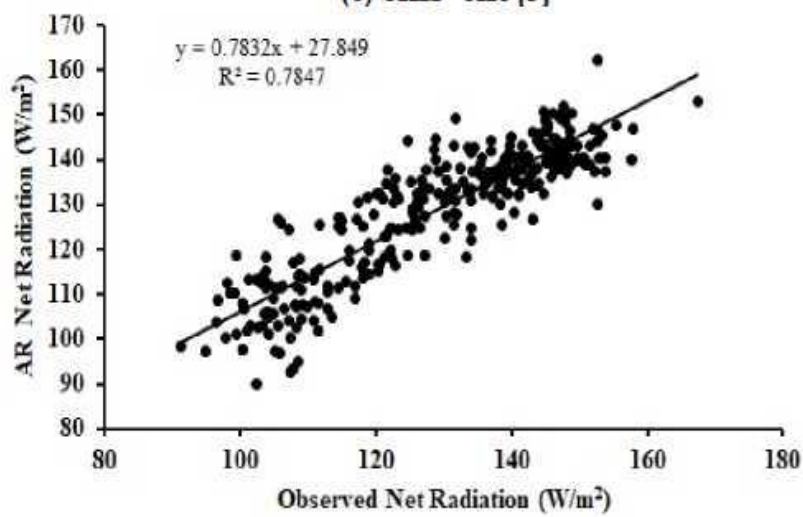

(b) SHH - AR $\{2\}$

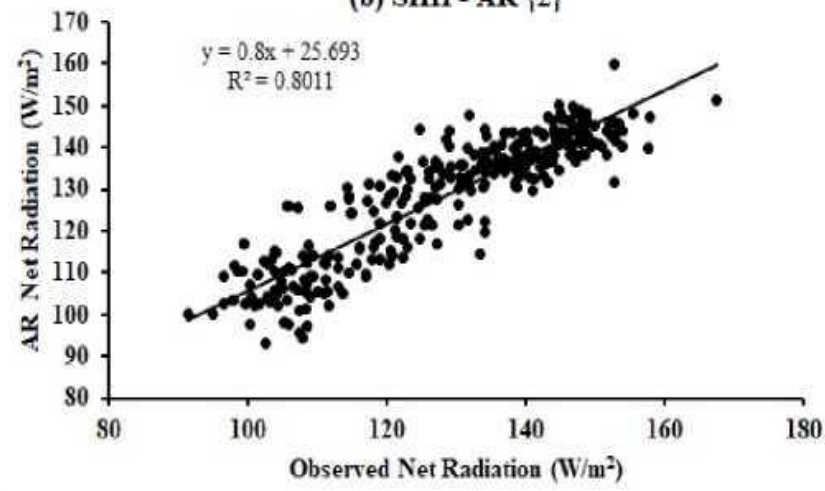

(d) SHH - AR \{4\}

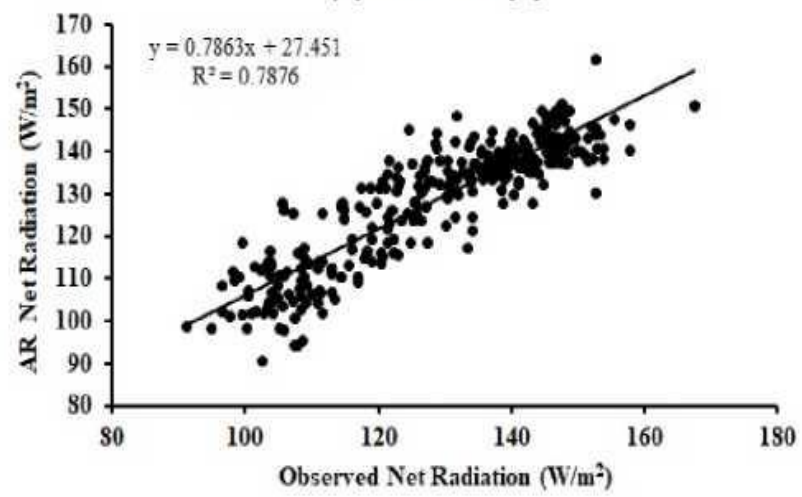

Fig. 4 Scatter plots of the observed net radiation and AR net radiation over SHH zone in Nigeria

(a) Humid Region - AR \{1\}

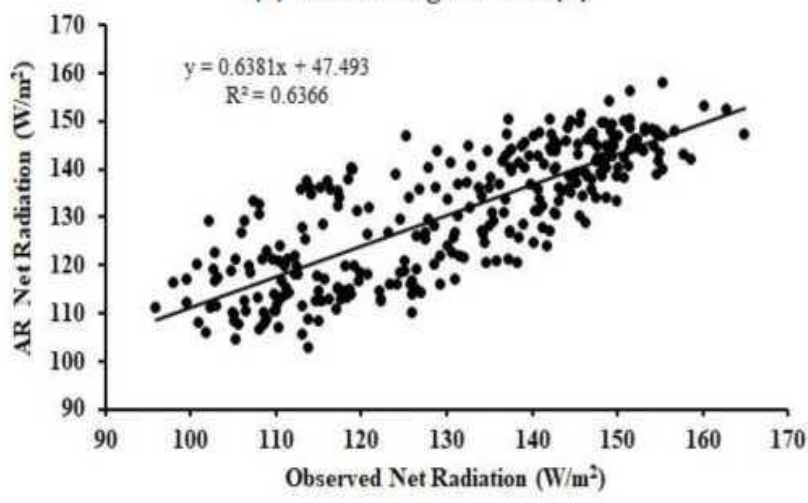

(c) Humid Region - AR \{3\}

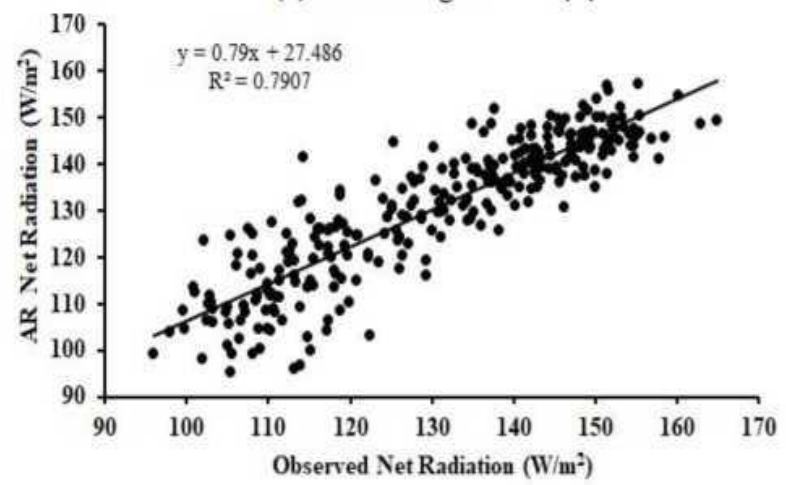

(b) Humid Region - AR \{2\}

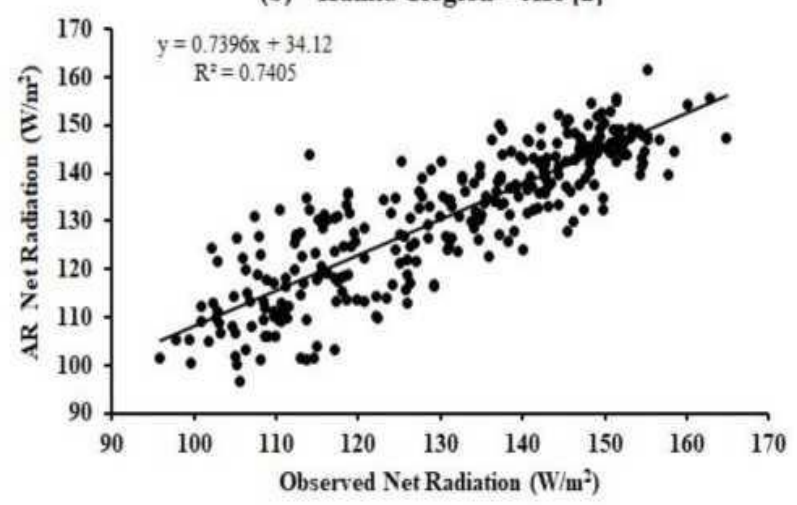

(d) Humid Region - AR \{4\}

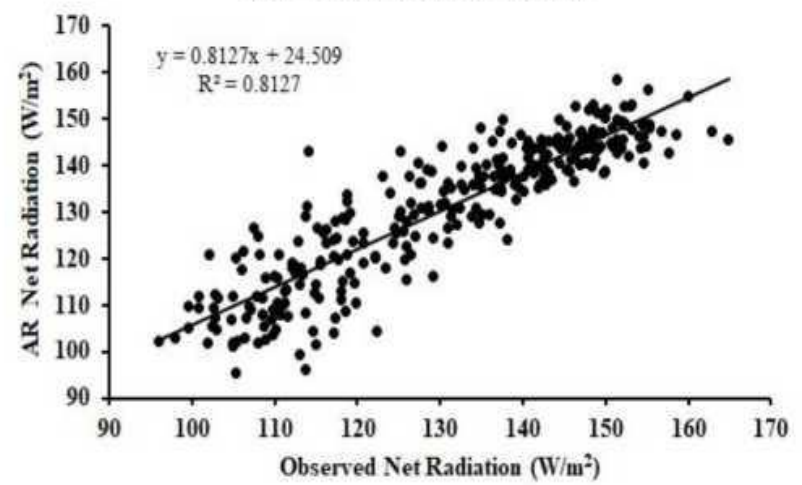

Fig. 5 Scatter plots of the observed net radiation and AR net radiation over HUM zone in Nigeria 
Figs. 6 - 9 show the variograms of observed net radiation with $A R\{p\}$ estimated

(a) Semi Arid - AR $\{1\}$

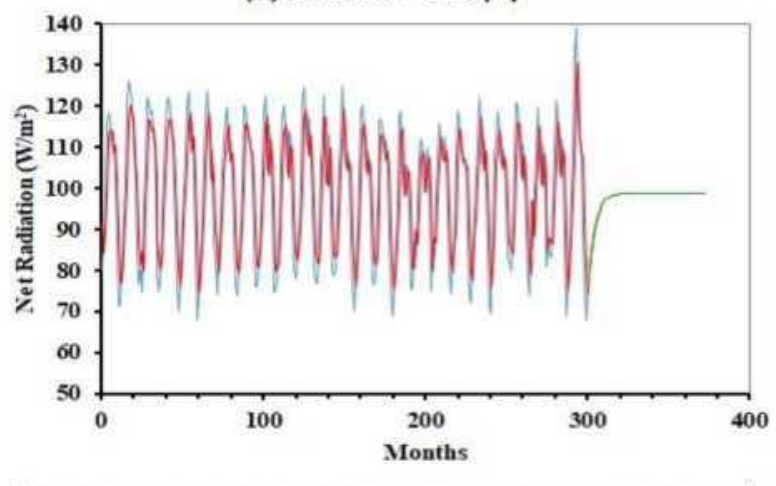

- Observed Net Radiation —AR Net Radiation — Prediction

(c) Semi Arid - AR \{3\}

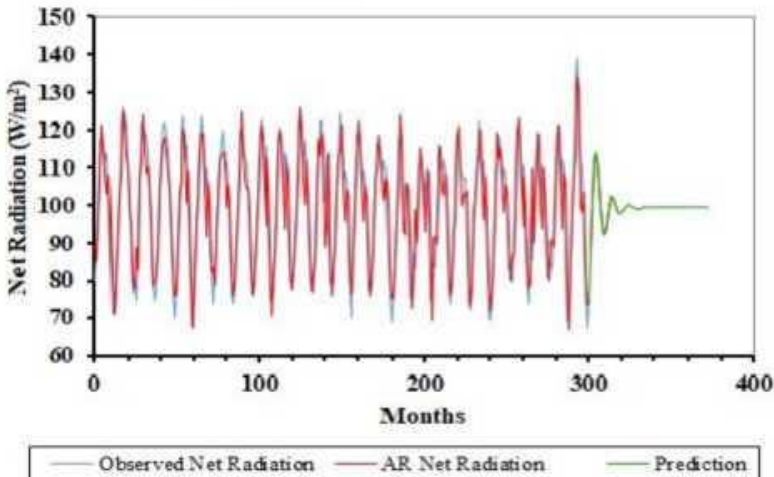

net radiation over four climatic regions in Nigeria.

(b) Semi Arid - AR \{2\}

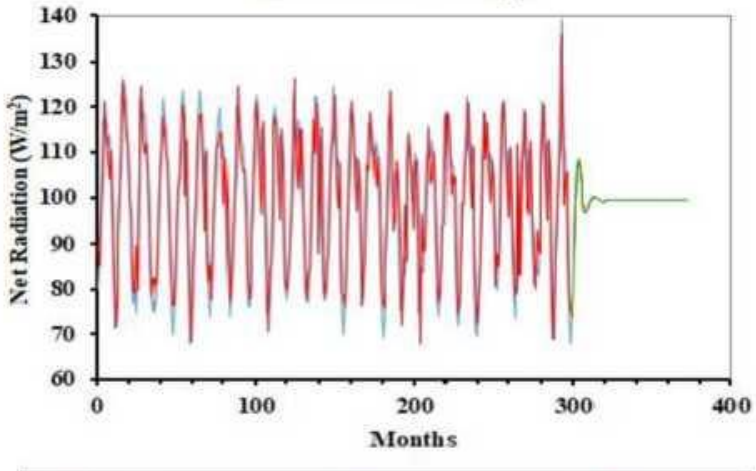

Observed Net Radation —AR Net Radiation - Prediction

(d) Semi Arid - AR \{4\}

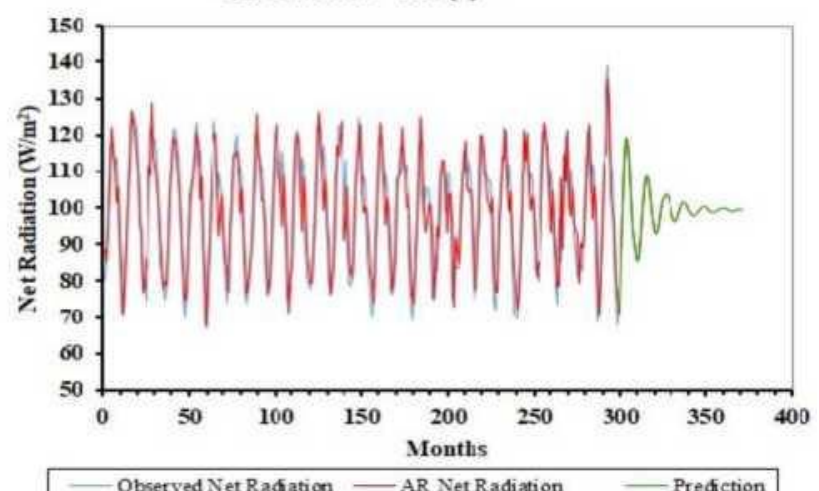

Fig. 6 Variation of the observed net radiation with AR predicted net radiation over SAR region in Nigeria

(a) SHD - AR $\{1\}$

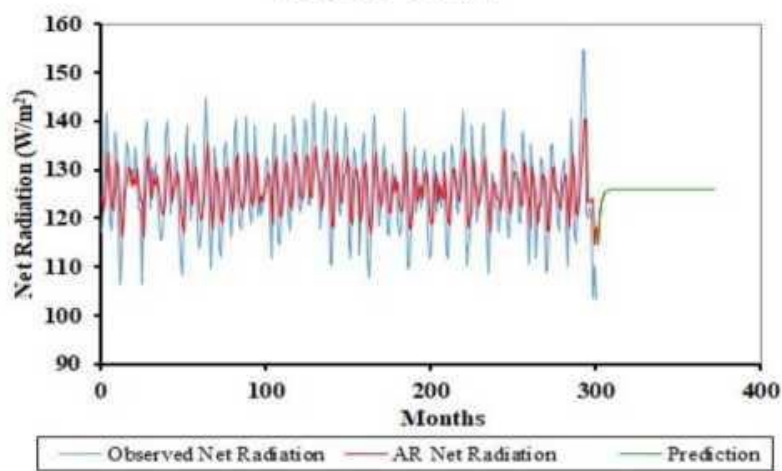

(c) SHD - AR $\{3\}$

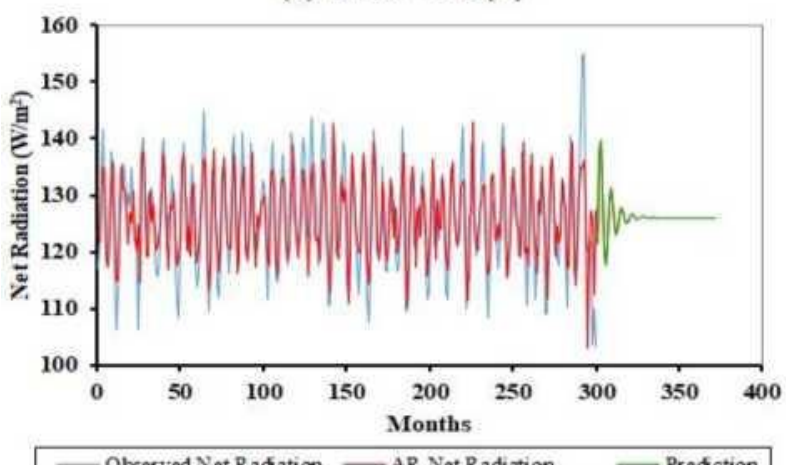

(b) SHD - AR $\{2\}$

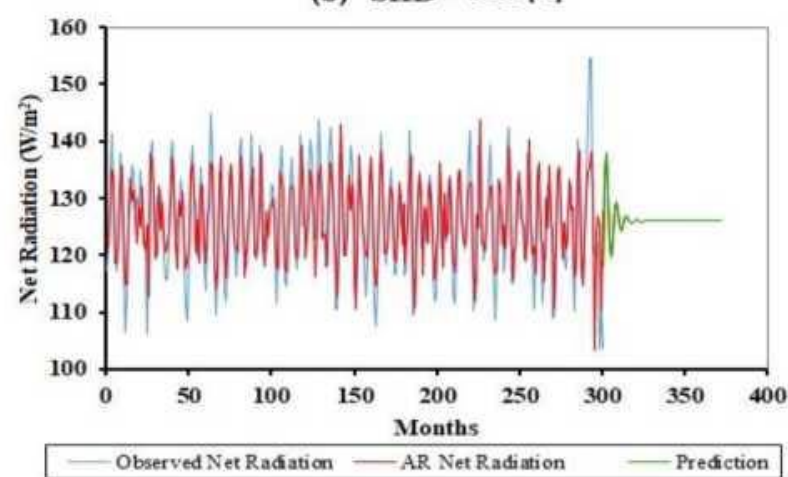

(d) SHD - AR $\{4\}$

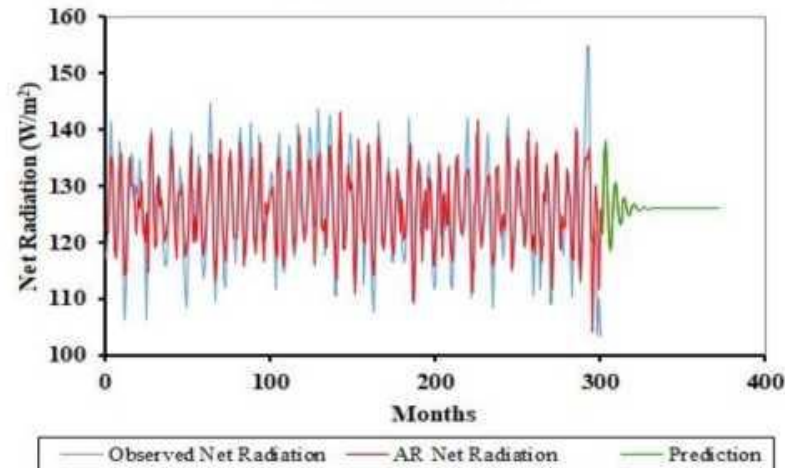

Fig. 7 Variation of the observed net radiation with AR predicted net radiation over SHD region in Nigeria 
(a) SHH - AR $\{1\}$

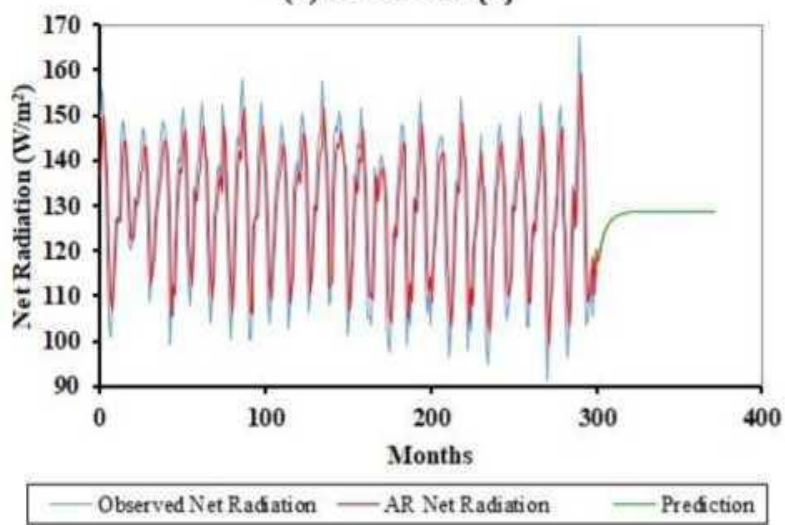

(c) SHH - AR \{3\}

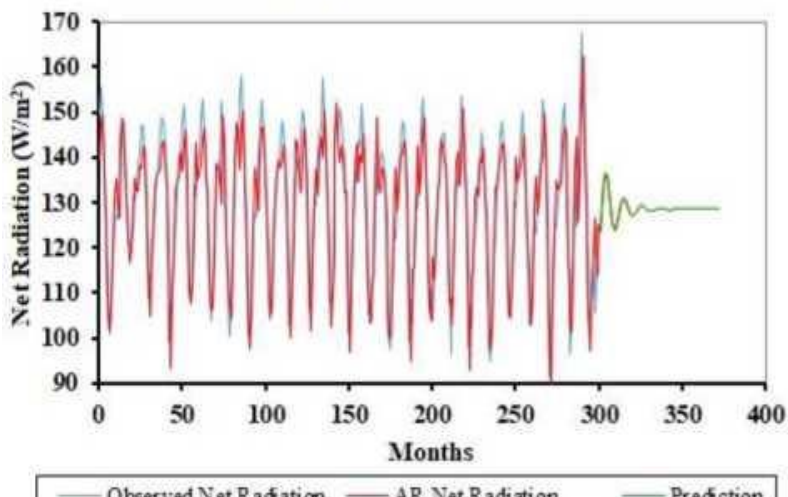

(b) SHH - AR \{2\}

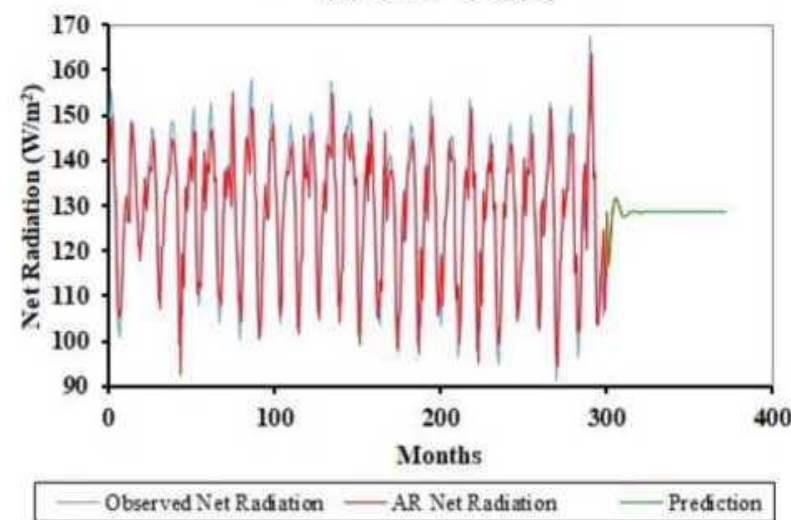

(d) SHH - AR $\{4\}$

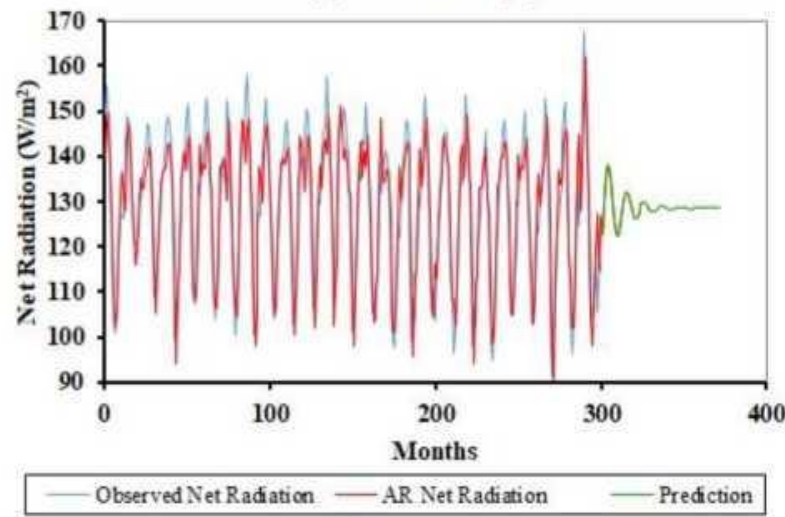

Fig. 8 Variation of the observed net radiation with AR predicted net radiation over SHH region in Nigeria

(a) Humid Region - AR \{1\}

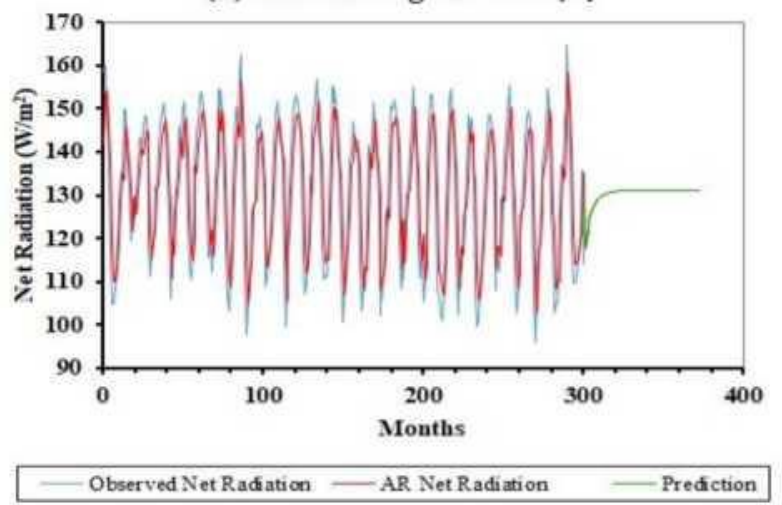

(c) Humid Region - AR \{3\}

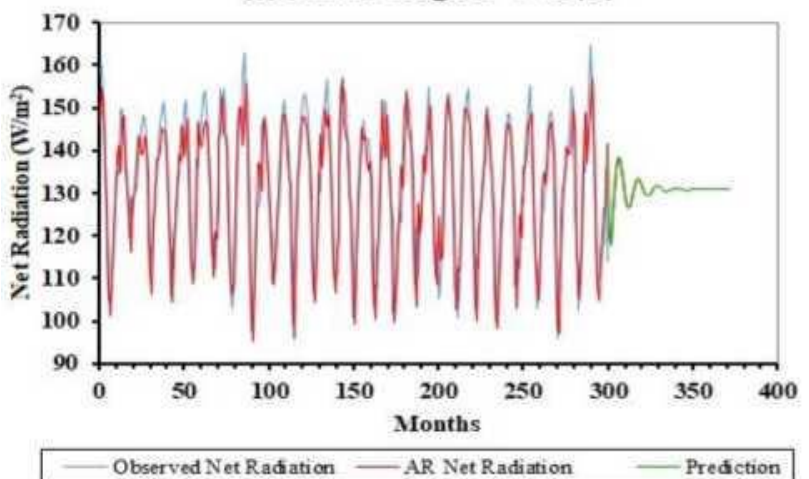

(b) Humid Region - AR \{2\}

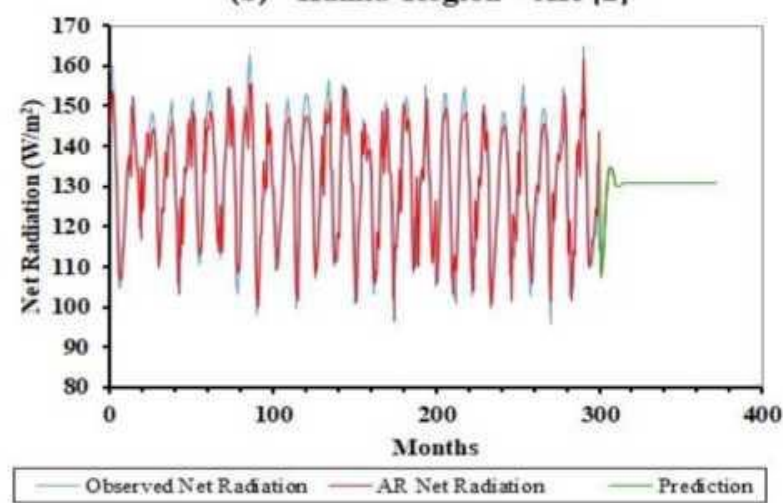

(d) Humid Region - AR \{4\}

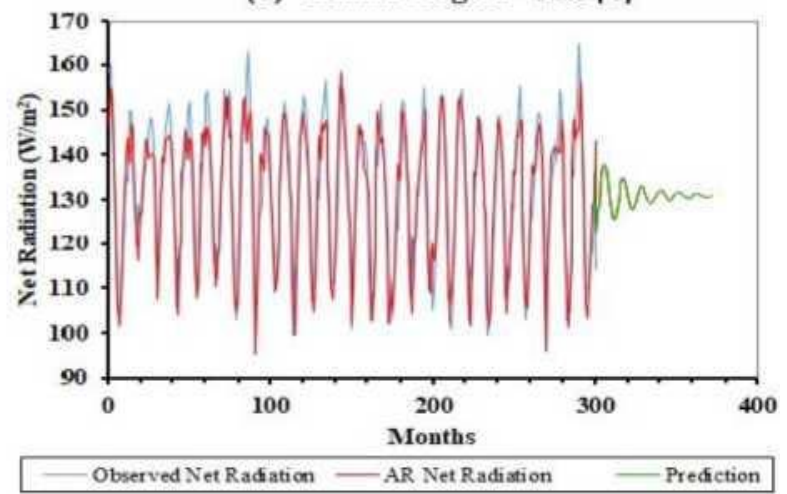

Fig. 9 Variation of the observed net radiation with AR predicted net radiation over HUM region in Nigeria 
In all the figures $(6-9)$, variations between observed and $\operatorname{AR}\{\mathrm{p}\}$ net radiation showed similar patterns and they were also closely monitored to each other. The future prediction made by the AR $\{\mathrm{p}\}$ model for 72 next months (2008 - 2013) showed that they would be decreased in the magnitude of net radiation in agreement with recommendation in [28].

\section{Conclusion}

Autoregressive models with four different orders were developed to deduce the net radiation over four climatic regions in Nigeria using in situ data from NIMET, Lagos. The parameter estimates of each of the models were obtained using XLSTAT software (2016 version) to develop the models for the four regions and some of their selected stations over Nigeria. The developed models were used to predict the net radiation for the period of 72 months (2008 - 2013). The predicted values of net radiation were compared with the observed values using the scattergrams and variograms with correlation equations. Analyses showed that the autoregressive models of the fourth order (AR $\{4\}$ ) gave maximum values of the coefficient of determination of 0.8127 in the HUM, 0.7876 in the SHH, 0.5765 in the SHD and 0.7973 in the SAR regions. The performance of other metrics such as the mean squared errors, root mean square errors, mean absolute percentage error and the Akaike's information criterion gave good values for all models especially for $A R\{4\}$. It can be established that the AR model of higher orders will give an accurate estimation of net radiation in any location in Nigeria. That is, the autoregressive $(A R\{p\})$ models will be a good model for the estimation of net radiation to a high degree of accuracy at higher orders.

\section{Conflict of Interests}

The author declares that there is no conflict of interests regarding the publication of this paper.

\section{References}

[1] B. Wu, S. Liu, W. Zhu, N. Yan, Q. Xing, and S. Tan, "An improved approach for estimating daily net radiation over the Heihe River Basin," Sensors, vol. 17 , no. 1 , p. 86,2017 , doi: 10.3390/s17010086

[2] E. D. P. Lima, G. C. Sediyama, B. B. d. Silva, J. M. Gleriani, and V. P. Soares, "Seasonality of net radiation in two sub-basins of Paracatu by the use of MODIS sensor products," Engenharia Agricola, vol. 32, no. 6, pp. 1184-1196, 2012.

[3] Z. Li, F. L. Zheng, and W.-Z. Liu, "Spatiotemporal characteristics of reference evapotranspiration during 1961-2009 and its projected changes during 2011-2099 on the Loess Plateau of China," Agricultur. Forest Meteorol., vol. 154, pp. 147-155, 2012.

[4] X.-M. Zhou, B.-H. Tang, H. Wu, and Z.-L. Li, "Estimating net surface longwave radiation from net surface shortwave radiation for cloudy skies," Int. J. Remote Sensing, vol. 34, no. 22, pp. 8104-8117, 2013, doi: 10.1080/01431161.2013.832002

[5] V. Ramanathan, "The role of earth radiation budget studies in climate and general circulation research," J. Geophysical Res. Atmospheres, vol. 92, D4, pp. 4075-4095, 1987.

[6] J. T. Suttles, and G. Ohring, "Surface radiation budget for climate applications," vol. 1169, pp. 1-123, 1986.

[7] F. Golkar, W. B. Rossow, and A. A. Sabziparvar, "Refining surface net radiation estimates in arid and semi-arid climates of Iran," Advan. Space Res., vol. 61, no. 12, pp. 2932-2941, 2018, doi: 10.1016/j.asr.2018.03.026

[8] S. V. S. Sai Krishna, P. Manavalan, and P. V. N. Rao, "Estimation of net radiation using satellite based data inputs," Int. Arch. Photogramm. Remote Sens. Spatial Inf. Sci., XL-8, pp. 307313, 2014, doi: 10.5194/isprsarchives-XL-8307-2014

[9] M. Mira et al., "Uncertainty assessment of surface net radiation derived from Landsat images," Remote Sensing of Environment, vol. 175, pp. 251-270, 2016, doi: $10.1016 /$ j.rse.2015.12.054

[10] B. Jiang et al., "Empirical estimation of daytime net radiation from shortwave radiation and ancillary information," Agricultur. Forest Meteorol., vol. 211, pp. 23-36, 2015.

[11] R. Blaga, A. Sabadus, N. Stefu, C. Dughir, M. Paulescu, and V. Badescu, "A current perspective on the accuracy of incoming solar energy forecasting," Progress Energy Combust. Sci., vol. 70, pp. 119-144, 2019.

[12] R. J. Hyndman, and A. B. Koehler, "Another look at measures of forecast accuracy," International J. Forecasting, vol. 22, no. 4, pp. 679-688, 2006.

[13] O. S. Ojo, and O. O. Omitusa, "Diurnal analysis of decadal changes in some climate variables over semi-arid and humid zones in Nigeria," Int. J. Recent Innov. Appl. Physics Applicat., vol. 1, no. 1, 2018.

[14] P. J. Brockwell, and R. A. Davis, Introduction to time series and forecasting. New York: springer, 2016.

[15] S. Bali, B. Hazra, and V. Pakrashi, "Quantile autoregressive modeling for non-linear change detection in vibrating structural systems," Mechanics Research Communications, vol. 100, p. 103397, 2019. 
[16] M. S. Okundamiya, J. O. Emagbetere, and E. A. Ogujor, "Evaluation of various global solar radiation models for Nigeria," Int. J. Green Energy, vol. 13, no. 5, pp. 505-512, 2016, doi: $10.1080 / 15435075.2014 .968921$

[17] J. Huang, M. Korolkiewicz, M. Agrawal, and J. Boland, "Forecasting solar radiation on an hourly time scale using a coupled autoregressive and dynamical system model," Sol. Energy, vol. 87, pp. 136-149, 2013, doi: 10.1016/j.solener.2012.10.012

[18] A. Bhargawa, and A. K. Singh, "Solar irradiance, climatic indicators and climate change - An empirical analysis," Advan. Space Res., vol. 64, no. 1 , pp. 271-277, 2019, doi: 10.1016/j.asr.2019.03.018

[19] André, S. Dabo-Niang, T. Soubdhan, and H. Ould-Baba, "Predictive spatio-temporal model for spatially sparse global solar radiation data," Energy, vol. 111, pp. 599-608, 2016.

[20] C. Voyant, J. G. de Gooijer, and G. Notton, "Periodic autoregressive forecasting of global solar irradiation without knowledge-based model implementation," Sol. Energy, vol. 174, pp. 121-129, 2018.

[21] G. Chattopadhyay and S. Chattopadhyay, "Autoregressive forecast of monthly total ozone concentration: A neurocomputing approach," Computers \& Geosciences, vol. 35, no. 9, pp. 1925-1932, 2009, doi: 10.1016/j.cageo.2008.11.007

[22] H. Chiri et al., "Statistical simulation of ocean current patterns using autoregressive logistic regression models: A case study in the Gulf of
Mexico," Ocean Modelling, vol. 136, pp. 1-12, 2019, doi: 10.1016/j.ocemod.2019.02.010

[23] J. L. Devore, Probability and Statistics for Engineering and the Sciences: Cengage learning, 2011.

[24] C. J. Willmott, and K. Matsuura, "On the use of dimensioned measures of error to evaluate the performance of spatial interpolators," Int. J. Geographical Information Sci., vol. 20, no. 1, pp. 89-102, 2006, doi: 10.1080/13658810500286976

[25] B. Adeyemi, and O. S. Ojo, "Empirical modeling of solar radiation for selected cities in Nigeria using multivariate regression technique," African J. Environmental Sci. Technol., vol. 8, no. 10, pp. 582-595, 2014.

[26] M. Snipes, and D. C. Taylor, "Model selection and Akaike Information Criteria: An example from wine ratings and prices," Wine Economics and Policy, vol. 3, no. 1, pp. 3-9, 2014, doi: 10.1016/j.wep.2014.03.001

[27] O. S. Ojo, and B. Adeyemi, "Estimation of solar radiation using air temperature and geographical coordinate over Nigeria," Pacific $J$. Sci. Technol., vol. 15, no. 2, pp. 78-88, 2014.

[28] O. S. Ojo, B. Adeyemi, and E. O. Ogolo, "Assessments of the night-time and daytime radiative fluxes balance on seasonal timescale over West Africa," J. Atmospher. SolarTerrestrial Physics, vol. 191, p. 105048, 2019, doi: 10.1016/j.jastp.2019.05.012 